\title{
MULTIPLIER SPACES FOR THE MORTAR FINITE ELEMENT METHOD IN THREE DIMENSIONS
}

\author{
CHISUP KIM, RAYTCHO D. LAZAROV, JOSEPH E. PASCIAK, AND \\ PANAYOT S. VASSILEVSKI
}

\begin{abstract}
We consider the construction of multiplier spaces for use with the mortar finite element method in three spatial dimensions. Abstract conditions are given for the multiplier spaces which are sufficient to guarantee a stable and convergent mortar approximation. Three examples of multipliers satisfying these conditions are given. The first one is a dual basis example while the remaining two are based on finite volumes. Finally, the results of computational examples illustrating the theory are reported.
\end{abstract}

\section{INTRODUCTION}

Domain decomposition methods have been widely used to design parallel algorithms for solving partial differential equations. The main idea of such methods as is well-known is the following. The boundary value problem posed on a given domain is discretized by finite elements, finite differences, spectral or other approximation methods and as a result an algebraic problem is obtained. Preconditioners that can utilize parallel computer architectures are based on splitting the original problem into a number of subproblems with subsequent subproblem solutions and iteration over the unknowns on the subdomain interfaces. This approach has been extensively studied in the last two decades (see, e.g., $[18,19,24,25])$. Often this approach is referred to as a conforming domain decomposition method.

The rapid growth in the demand for large scale simulations and the proliferation of CAD/CAM systems in the last decade led to the necessity for different research teams to interact and use various computing environments and tools for solving complex phenomena. Such interactions have resulted in the design of a new class of domain decomposition methods, often called nonconforming or mortar methods. In contrast with the conforming domain decomposition method, the subdomains now can be meshed independently, that is, in general, the grids do not match across the subdomain interfaces. The mortar method provides an approach to glue together the approximations on the subdomains by imposing, in a weak sense, the continuity of the solution across these interfaces. Since the introduction of the mortar method as a coupling technique for spectral and finite element approximations (see, e.g., $[8,9,10,22]$ ), it has become a very successful technique

Date: August 28, 2000.

1991 Mathematics Subject Classification. 65F10, 65N30.

Key words and phrases. mortar, finite element method, Lagrange multipliers, domain decomposition.

This work was supported by the National Science Foundation under grant DMS 9973328, the Environmental Protection Agency under grant R 825207 and the State of Texas under ARP/ATP grant 010366-168. The work of the last author was performed under the auspices of the U.S. Department of Energy by Lawrence Livermore National Laboratory under Contract W-7405-Eng-48. 
for non-matching grids yielding a stable and optimally convergent global approximation. The research in this approach has been motivated by the flexibility of the method and by its potential for efficient parallel implementation.

The mortar finite element method has been studied in $[5,6,8,9]$, where optimal order convergence in $H^{1}$-norm was established. Three-dimensional mortar finite element analysis has been given in [6] and the $h-p$ version has been studied in [32]. Mortar mixed finite element approximations for second order elliptic problems have been discussed in [4] and mortar methods for finite volume method approximations are presented in [23].

The mortar approximations involve constraints, namely the weak continuity, on the space. These constraints could be treated as Lagrange multipliers (see, e.g., [5]), leading to a saddle point problem, which is symmetric and indefinite. On the other hand, it is also possible to view the mortar problem as a non-conforming finite element approximation. This approach leads to a symmetric positive definite problem (see, e.g., [7, 26]). In our analysis, we consider the latter approach. In either case, efficient iterative methods are essential for the overall performance of the method. Multigrid/multilevel preconditioners for the mortar finite element approximations have been studied in $[12,17,26,27]$ while preconditioners based on substructuring have been studied in [1, 2, 29].

The continuity of the solution across the subdomain interfaces is imposed in a weak sense by using the multiplier space. The resulting multiplier approximates the trace of the co-normal derivative of the solution at the subdomain interface. As the multiplier most naturally belongs to a negative Sobolev space, continuity of the functions in the mortar approximation subspaces is not necessary. However, most of the finite element approximations of the mortar space used in the mortar finite element method have been related to the traces of the finite element spaces on the interfaces, which results in continuous functions. Some instances of discontinuous mortar spaces have been considered in $[4,29,33]$. One approach used to construct these spaces is based on the dual bases and has several important computational advantages compared with continuous mortar functions. Specifically, the resulting mass matrix is diagonal and so its inversion is trivial.

In this paper we construct three different mortar spaces in three dimensions; one based on the dual basis approach and two additional examples based on finite volume approaches. They all involve discontinuous functions and lead to relatively simple constructions. The dual basis example is the most interesting. As mentioned above, the mass matrix is diagonal and so the non-conforming basis elements have local support. In addition, we will show that this method remains stable and convergent even in the presence of mesh refinement provided that the meshes are locally quasi-uniform and that the triangulations align on the boundaries of the subdomain interfaces. In contrast, stability of the mortar method with continuous multipliers requires additional conditions on the mesh (see [32] for the case of one dimensional interfaces). These additional conditions are related to the stability of the elliptic projection in $L^{2}$ and have been studied in $[13,16,21]$ although in a different context.

We provide the construction and stability analysis of the mortar spaces via a set of abstract conditions which are later verified for our particular examples. These conditions are general enough to handle the general mesh refinement dual basis example. For completeness, we also provide an error analysis of the method based on these conditions. 
Other work on the dual basis Lagrange multipliers was done concurrently with that of this paper [34]. There they analyze a similar method as the dual basis example considered in this paper using mesh dependent weighted norms under the assumption of a globally quasi-uniform triangulation. They also consider multigrid methods for solving the resulting systems of algebraic equations.

The remainder of the paper is organized as follows. In Section 2, we introduce the mortar finite element approximation of the Poisson equation with homogeneous Dirichlet boundary condition. The abstract conditions on the multiplier spaces are formulated in Section 3 and the error analysis of the method is presented. Three examples of mortar spaces follow in Section 4. Finally, the results of numerical experiments are presented in Section 5.

\section{Problem Formulation AND NOtATion}

We consider the Dirichlet problem on a bounded polyhedral domain $\Omega$ in $\mathbb{R}^{3}$. Given $f \in L^{2}(\Omega)$, we want to approximate the solution $u \in H_{0}^{1}(\Omega)$ of

$$
\begin{aligned}
-\Delta u=f & \text { in } \Omega, \\
u=0 & \text { on } \partial \Omega .
\end{aligned}
$$

Extensions to more general second order elliptic partial differential equations and systems and to more general boundary conditions are possible and demonstrated in Section 5 .

The domain $\Omega$ is partitioned into $K$ non-overlapping polyhedral subdomains $\Omega_{i}, i=$ $1, \ldots, K$, that is,

$$
\bar{\Omega}=\bigcup_{i=1}^{K} \overline{\Omega_{i}}, \quad \text { with } \Omega_{i} \cap \Omega_{j}=\emptyset \text { for } i \neq j .
$$

It is assumed that each subdomain $\Omega_{i}$ is associated with a locally quasi-uniform triangulation $\mathcal{T}_{i}$ of tetrahedra. We denote by $\bar{h}_{i}$ the mesh size of $\mathcal{T}_{i}$. In other words, $\bar{h}_{i}$ is the maximum of the diameters of the tetrahedra in the mesh $\mathcal{T}_{i}$. The triangulations in the subdomains are independent of each other.

To describe the subdomain interfaces, we define a set $\mathcal{I}$ by

$$
\mathcal{I}=\left\{\begin{array}{l|l}
i j & \begin{array}{l}
1 \leq i, j \leq K \\
\partial \Omega_{i} \cap \partial \Omega_{j} \text { is a two-dimensional domain } \\
\text { and } j i \notin \mathcal{I}
\end{array}
\end{array}\right\}
$$

For each pair $i j \in \mathcal{I}$, we define $\Gamma_{i j}=\partial \Omega_{i} \cap \partial \Omega_{j}$ to be the interface between the mortar subdomain $\Omega_{i}$ and the non-mortar subdomain $\Omega_{j}$. The triangulation on an interface $\Gamma_{i j}$ is denoted by $\mathcal{T}_{i j}$. This triangulation is inherited from that of the non-mortar subdomain $\Omega_{j}$, namely $\mathcal{T}_{j}$.

We now discuss the conditions on the subdomain partition and the triangulation. To begin with, we do not require the subdomains to align. In other words, we allow a single face of a polyhedral subdomain to have non-empty intersections with faces from more than one of the remaining subdomains. We do, however, require that the triangulations align with the subdomain partition. That is, if a face of a tetrahedron in a triangulation 


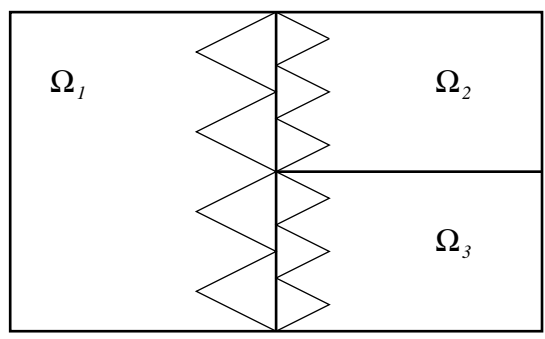

Figure 1. An example of a two-dimensional domain with 3 subdomains. The subdomains do not align but the triangulation does.
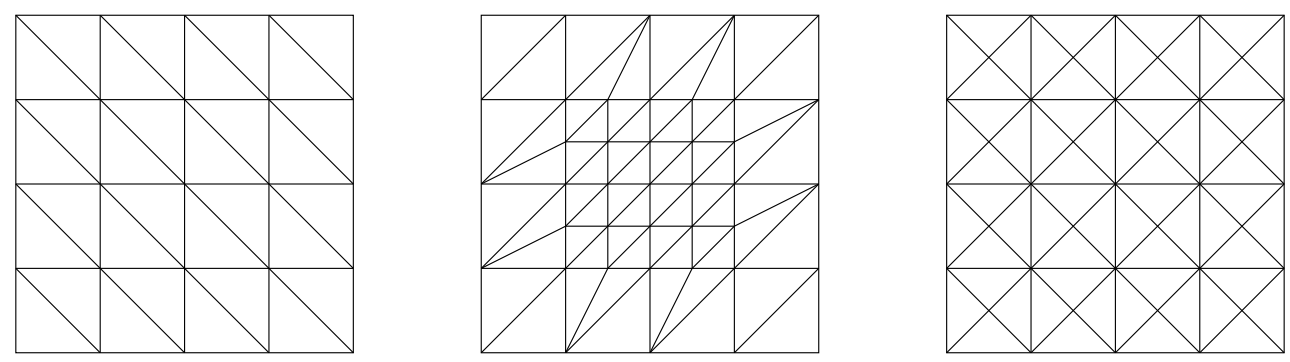

FiguRE 2. Examples of triangulations for an interface between threedimensional subdomains. The triangulations all match on the boundary of the interface.

$\mathcal{T}_{i}$ or $\mathcal{T}_{j}$ intersects an interface $\Gamma_{i j}$, then it must be completely contained in $\Gamma_{i j}$. A twodimensional domain with non-aligning subdomain partition and aligning triangulation is shown in Figure 1. In the analysis, we will also need the following condition.

(M.1) The subdomain triangulations match on interface boundaries.

This condition is readily met in the two-dimensional case, where an interface boundary degenerates into isolated points. In three-dimensions, as the examples in Figure 2 suggest, this is not too strict a restriction on the triangulation, although not a condition as easily satisfied as in the two-dimensional case. These conditions could be relaxed in the special case when the non-mortar mesh satisfies an inverse inequality (see Remark 3.2 and [34]). This case of non-aligning triangulation is also of interest since then there would be fewer restrictions in the meshing process in each subdomain.

Next, we consider the mortar finite element space. For the sake of simplicity, piecewise linear finite element spaces will be used. Our theory, however, generalizes to higher order finite element spaces without difficulty. Define, for each $i=1, \ldots, K$, the finite element space $X_{h, i}$ in the subdomain $\Omega_{i}$ by

$$
X_{h, i}=\left\{\begin{array}{l}
v \\
\begin{array}{l}
v \text { is linear on each tetrahedron in } \mathcal{T}_{i}, \\
v \text { is continuous on } \Omega_{i}, \\
\text { and } v=0 \text { on } \partial \Omega \cap \partial \Omega_{i}
\end{array}
\end{array}\right\}
$$

and the unconstrained global space $\widetilde{X}_{h}$ by

$$
\widetilde{X}_{h}=\left\{v|v|_{\Omega_{i}} \in X_{h, i} \text { for all } i=1, \ldots, K\right\} .
$$


The mortar finite element method is a non-conforming finite element method. Since the $L^{2}$-trace of the solution of (2.1) is continuous, some type of continuity must be imposed on the space $\widetilde{X}_{h}$. However, the meshes defining $X_{h, i}$ and $X_{h, j}$ do not necessarily align on $\Gamma_{i j}$. Thus, to retain the approximation properties on the interfaces, one can only impose continuity weakly. To this end, we introduce the multiplier spaces $M_{h}\left(\Gamma_{i j}\right)$ for each $i j \in \mathcal{I}$ and define the mortar finite element space $X_{h}$ by

$$
X_{h}=\left\{v \in \widetilde{X}_{h} \mid \int_{\Gamma_{i j}}[v]_{i j} \varphi d s=0 \text { for all } \varphi \in M_{h}\left(\Gamma_{i j}\right) \text { and all } i j \in \mathcal{I}\right\} .
$$

Here, $[v]_{i j}=\left.\left(\left.v\right|_{\Omega_{i}}\right)\right|_{\Gamma_{i j}}-\left.\left(\left.v\right|_{\Omega_{j}}\right)\right|_{\Gamma_{i j}}$. The multiplier space $M_{h}\left(\Gamma_{i j}\right)$ will be defined in terms of the triangulation $\mathcal{T}_{i j}$ inherited from that of the non-mortar subdomain. It is the purpose of this paper to formulate abstract conditions and examples for these spaces which give rise to stable finite element approximations. The mortar finite element problem is now formulated as follows.

Find $u_{h} \in X_{h}$ such that

$$
\widetilde{A}\left(u_{h}, v\right)=(f, v) \quad \text { for all } v \in X_{h},
$$

where

$$
\widetilde{A}(u, v)=\sum_{i=1}^{K} \int_{\Omega_{i}} \nabla u \cdot \nabla v d x
$$

and

$$
(f, v)=\int_{\Omega} f v d x
$$

In the rest of this section, we set up additional notation which will be used in this paper. We will denote by $C$ and $c$ generic positive constants. These constants take on different values in different occurrences but are always independent of the mesh parameters. The Sobolev space $H^{k}(\Omega)$, for a non-negative integer $k$, is the set of functions in $L^{2}(\Omega)$ whose weak derivatives of order up to $k$ are also in $L^{2}(\Omega)$ (see, e.g., [15, 28]). For real $s$ with $k<s<k+1$ for some non-negative integer $k, H^{s}(\Omega)$ is defined by interpolation (e.g. using the real method) between $H^{k}(\Omega)$ and $H^{k+1}(\Omega)$ (see, e.g., [31]). There is a special interpolation space which will play an important role in the analysis of the mortar method. This space is obtained by interpolation between $L^{2}\left(\Gamma_{i j}\right)$ and $H_{0}^{1}\left(\Gamma_{i j}\right)$, and is denoted by $H_{00}^{1 / 2}\left(\Gamma_{i j}\right)$. As usual, $\|\cdot\|_{s}$ and $|\cdot|_{s}$ denote the $H^{s}(\Omega)$ norm and seminorm. If we denote by $D$ a subdomain $\Omega_{i}$ or an interface $\Gamma_{i j}$, the $H^{s}(D)$ norm and seminorm will be written $\|\cdot\|_{s, D}$ and $|\cdot|_{s, D}$ respectively. This convention applies also to the $L^{2}(D)$ inner product, which will be denoted by $(\cdot, \cdot)_{D}$. We define the norm $\|\cdot \cdot\|$ by

$$
\|u\|^{2}=\sum_{i=1}^{K}\|u\|_{1, \Omega_{i}}^{2} .
$$

We shall also use the following spaces. Let

$$
S_{h}^{0}\left(\Gamma_{i j}\right)=S_{h}\left(\Gamma_{i j}\right) \cap H_{0}^{1}\left(\Gamma_{i j}\right)
$$

where

$$
S_{h}\left(\Gamma_{i j}\right)=\left\{v|v=w|_{\Gamma_{i j}} \text { for some } w \in X_{h, j}\right\} .
$$


Finally, for each $i j \in \mathcal{I}$, the mortar projection $\Pi_{i j}: L^{2}\left(\Gamma_{i j}\right) \rightarrow S_{h}^{0}\left(\Gamma_{i j}\right)$ is defined by

$$
\left(\Pi_{i j} u, \varphi\right)_{\Gamma_{i j}}=(u, \varphi)_{\Gamma_{i j}} \quad \text { for all } \varphi \in M_{h}\left(\Gamma_{i j}\right) .
$$

This operator was used in $[6,7,32]$ and plays a central role in the analysis of the mortar finite element method.

\section{Abstract multiplier CONDITIONS AND ERROR ANALYSiS}

We start this section by giving some abstract conditions for the multiplier spaces which guarantee a stable and convergent mortar finite element method. We introduce the following properties for the multiplier spaces:

(A.1) For each $i j \in \mathcal{I}, M_{h}\left(\Gamma_{i j}\right)$ contains constant functions.

(A.2) For each $i j \in \mathcal{I}, S_{h}^{0}\left(\Gamma_{i j}\right)$ and $M_{h}\left(\Gamma_{i j}\right)$ have the same dimension.

(A.3) There is a constant $C$ not depending on the triangulation or $i j \in \mathcal{I}$ such that

$$
\|\theta\|_{0, \Gamma_{i j}} \leq C \sup _{\psi \in M_{h}\left(\Gamma_{i j}\right)} \frac{(\theta, \psi)_{\Gamma_{i j}}}{\|\psi\|_{0, \Gamma_{i j}}},
$$

for all $\theta \in S_{h}^{0}\left(\Gamma_{i j}\right)$ and $i j \in \mathcal{I}$.

(A.4) There is a constant $C$ not depending on the triangulation or $i j \in \mathcal{I}$ such that

$$
\inf _{\gamma \in M_{h}\left(\Gamma_{i j}\right)}\|\sigma-\gamma\|_{0, \Gamma_{i j}} \leq C \bar{h}_{j}\|\sigma\|_{1, \Gamma_{i j}}
$$

for all $\sigma \in H^{1}\left(\Gamma_{i j}\right)$ and $i j \in \Gamma_{i j}$.

We note that the following two inequalities are simple consequences of (A.4).

$$
\begin{aligned}
& \inf _{\gamma \in M_{h}\left(\Gamma_{i j}\right)}(\sigma-\gamma, \zeta)_{\Gamma_{i j}} \leq C \bar{h}_{j}\|\sigma\|_{1 / 2, \Gamma_{i j}}\|\zeta\|_{1 / 2, \Gamma_{i j}} \\
& \inf _{\gamma \in M_{h}\left(\Gamma_{i j}\right)} \sup _{\eta \in H^{1 / 2}\left(\Gamma_{i j}\right)} \frac{(\sigma-\gamma, \eta)_{\Gamma_{i j}}}{\|\eta\|_{1 / 2, \Gamma_{i j}}} \leq C \bar{h}_{j}\|\sigma\|_{1 / 2, \Gamma_{i j}}
\end{aligned}
$$

for all $\sigma, \zeta \in H^{1 / 2}\left(\Gamma_{i j}\right)$.

When every interface mesh $\mathcal{T}_{i j}$ is globally quasi-uniform, these conditions are sufficient for stable mortar finite element approximation (see Remark 3.1). We shall need an additional condition to handle the case when the mesh $\mathcal{T}_{i j}$ is only locally quasi-uniform. Let $\left\{\phi_{l} \mid l=1, \ldots, n\right\}$ denote the nodal basis for $S_{h}^{0}\left(\Gamma_{i j}\right)$. Given a function $\phi=\sum d_{l} \phi_{l} \in$ $S_{h}^{0}\left(\Gamma_{i j}\right)$, we define $\hat{\phi}=\sum h_{l}^{-1} d_{l} \phi_{l}$. Here $h_{l}$ is the local mesh size at the l'th node. To be precise, we can take $h_{l}$ to be the maximum of the diameters of the triangles which meet at the l'th vertex. Given $\psi \in M_{h}\left(\Gamma_{i j}\right)$, we then define $\hat{\psi} \in M_{h}\left(\Gamma_{i j}\right)$ by

$$
(\phi, \hat{\psi})_{\Gamma_{i j}}=(\hat{\phi}, \psi)_{\Gamma_{i j}} \quad \text { for all } \phi \in S_{h}^{0}\left(\Gamma_{i j}\right)
$$

It follows from (A.2) and (A.3) that there is a unique $\hat{\psi} \in M_{h}\left(\Gamma_{i j}\right)$ satisfying (3.2). When $\mathcal{T}_{i j}$ is only locally quasi-uniform, we use the following condition:

(A.5) There is a constant $C$ not depending on $i j \in \mathcal{I}$ or the triangulation such that

$$
\sum_{\tau \in \mathcal{T}_{i j}} h_{\tau}^{2}\|\hat{\psi}\|_{0, \tau}^{2} \leq C\|\psi\|_{0, \Gamma_{i j}}^{2} .
$$

Here, $h_{\tau}$ denotes the diameter of $\tau$. 
In general, this condition does not hold without further restriction on the triangulation. However, we will show that it holds for the dual basis example without any additional assumptions.

The next theorem provides an error analysis for the mortar method under the above conditions. For completeness, we include a proof and illustrate how (A.5) is applied in the analysis.

Theorem 3.1. Let $u$ and $u_{h}$ be the solutions for problems (2.1) and (2.2), respectively. Assume that $u \in H_{0}^{1}(\Omega)$ and $\left.u\right|_{\Omega_{i}} \in H^{2}\left(\Omega_{i}\right)$ for all $i=1, \ldots, K$. If the conditions (M.1) and (A.1)-(A.5) are satisfied, then there is a constant $C$ not depending on $\left\{\bar{h}_{i}\right\}_{i=1}^{K}$ such that

$$
\left\|u-u_{h}\right\|^{2} \leq C \sum_{i=1}^{K} \bar{h}_{i}^{2}\|u\|_{2, \Omega_{i}}^{2}
$$

For the proof of the theorem, we shall use two lemmas.

Lemma 3.1. Assume that the mesh $\mathcal{T}_{i j}$ on $\Gamma_{i j}$ is locally quasi-uniform and that (A.2), (A.3) and (A.5) hold. Then there is a constant $C$ not depending on mesh size or $i j \in \mathcal{I}$ satisfying

for all $u \in H_{00}^{1 / 2}\left(\Gamma_{i j}\right)$.

$$
\left\|\Pi_{i j} u\right\|_{H_{00}^{1 / 2}\left(\Gamma_{i j}\right)} \leq C\|u\|_{H_{00}^{1 / 2}\left(\Gamma_{i j}\right)}
$$

Proof. We need to verify that $\Pi_{i j}$ is stable in $L^{2}\left(\Gamma_{i j}\right)$ and $H_{0}^{1}\left(\Gamma_{i j}\right)$. Then, the result will follow from interpolation.

The proof of $L^{2}\left(\Gamma_{i j}\right)$ stability is standard. We observe that by (A.3), if $\theta \in S_{h}^{0}\left(\Gamma_{i j}\right)$ satisfies $(\theta, \psi)_{\Gamma_{i j}}=0$ for all $\psi$ in $M_{h}\left(\Gamma_{i j}\right)$ then $\theta=0$. This and (A.2) imply the unique solvability of (2.3). By (A.3),

$$
\left\|\Pi_{i j} w\right\|_{0, \Gamma_{i j}} \leq C \sup _{\psi \in M_{h}\left(\Gamma_{i j}\right)} \frac{\left(\Pi_{i j} w, \psi\right)_{\Gamma_{i j}}}{\|\psi\|_{0, \Gamma_{i j}}}=C \sup _{\psi \in M_{h}\left(\Gamma_{i j}\right)} \frac{(w, \psi)_{\Gamma_{i j}}}{\|\psi\|_{0, \Gamma_{i j}}} \leq C\|w\|_{0, \Gamma_{i j}} .
$$

Now we check the stability in $H_{0}^{1}\left(\Gamma_{i j}\right)$. Since the mesh is locally quasi-uniform, there is an operator $Q_{0}: L^{2}\left(\Gamma_{i j}\right) \rightarrow S_{h}^{0}\left(\Gamma_{i j}\right)$ satisfying (see, e.g., [20])

$$
\left\|Q_{0} u\right\|_{1, \Gamma_{i j}}^{2}+\sum_{\tau \in \mathcal{T}_{i j}} h_{\tau}^{-2}\left\|\left(I-Q_{0}\right) u\right\|_{0, \tau}^{2} \leq C\|u\|_{1, \Gamma_{i j}}^{2},
$$

for all $u \in H_{0}^{1}\left(\Gamma_{i j}\right)$. Fix $u \in H_{0}^{1}\left(\Gamma_{i j}\right)$. By (3.4) and triangle inequality, the lemma will follow if we show that

$$
\left\|\left(\Pi_{i j}-Q_{0}\right) u\right\|_{1, \Gamma_{i j}} \leq C\|u\|_{1, \Gamma_{i j}} .
$$

Let $\phi=\left(\Pi_{i j}-Q_{0}\right) u=\sum d_{l} \phi_{l}$. Then, by local inverse inequalities,

$$
\left\|\left(\Pi_{i j}-Q_{0}\right) u\right\|_{1, \Gamma_{i j}}^{2} \leq C \sum_{\tau \in \mathcal{T}_{i j}} h_{\tau}^{-2}\left\|\left(\Pi_{i j}-Q_{0}\right) u\right\|_{0, \tau}^{2} .
$$

We clearly have

$$
\int_{\tau} \phi^{2} d x \simeq h_{\tau}^{2}\left(d_{l_{1}}^{2}+d_{l_{2}}^{2}+d_{l_{3}}^{2}\right)
$$

where $\left\{l_{k}\right\}$ are the indices for the vertices of $\tau$. Here we used the notation $A \simeq B$ to mean that there are constants $c$ and $C$ not depending on the triangulation, functions in 
the subspaces defining $A$ and $B$, or $i j \in \Gamma_{i j}$ such that $c A \leq B \leq C A$. The constants $c$ and $C$ may depend on the minimal angle condition. By the local quasi-uniformity of $\mathcal{T}_{i j}$, it follows that

$$
\sum_{\tau \in \mathcal{T}_{i j}} h_{\tau}^{-2}\left\|\left(\Pi_{i j}-Q_{0}\right) u\right\|_{0, \tau}^{2} \simeq \sum_{l=1}^{n} d_{l}^{2} \simeq\|\hat{\phi}\|_{0, \Gamma_{i j}}^{2},
$$

where $\hat{\phi}$ is as in (3.2). Now, by (A.3), (3.4) and (A.5),

$$
\begin{aligned}
\|\hat{\phi}\|_{0, \Gamma_{i j}} & \leq C \sup _{\psi \in M_{h}\left(\Gamma_{i j}\right)} \frac{(\hat{\phi}, \psi)_{\Gamma_{i j}}}{\|\psi\|_{0, \Gamma_{i j}}}=C \sup _{\psi \in M_{h}\left(\Gamma_{i j}\right)} \frac{\left(\left(I-Q_{0}\right) u, \hat{\psi}\right)_{\Gamma_{i j}}}{\|\psi\|_{0, \Gamma_{i j}}} \\
& \leq C \sup _{\psi \in M_{h}\left(\Gamma_{i j}\right)} \frac{\left(\sum_{\tau \in \mathcal{T}_{i j}} h_{\tau}^{-2}\left\|\left(I-Q_{0}\right) u\right\|_{0, \tau}^{2}\right)^{1 / 2}\left(\sum_{\tau \in \mathcal{T}_{i j}} h_{\tau}^{2}\|\hat{\psi}\|_{0, \tau}^{2}\right)^{1 / 2}}{\|\psi\|_{0, \Gamma_{i j}}} \\
& \leq C\|u\|_{1, \Gamma_{i j}} .
\end{aligned}
$$

Combining the above inequalities (3.6), (3.7) and (3.8) establishes (3.5) and hence completes the proof of the lemma.

Remark 3.1. When $\mathcal{T}_{i j}$ is globally quasi-uniform, that is $h_{\tau} \geq c \bar{h}_{j}$ for all $\tau \in \mathcal{T}_{i j}$, the above lemma can be proved without condition (A.5). Under this condition, the argument following (3.6) can be simplified. By (A.3) and (3.4), for $u \in H_{0}^{1}\left(\Gamma_{i j}\right)$,

$$
\begin{aligned}
\left\|\left(\Pi_{i j}-Q_{0}\right) u\right\|_{0, \Gamma_{i j}} & \leq C \sup _{\psi \in M_{h}\left(\Gamma_{i j}\right)} \frac{\left(\left(\Pi_{i j}-Q_{0}\right) u, \psi\right)_{\Gamma_{i j}}}{\|\psi\|_{0, \Gamma_{i j}}} \\
& =C \sup _{\psi \in M_{h}\left(\Gamma_{i j}\right)} \frac{\left(\left(I-Q_{0}\right) u, \psi\right)_{\Gamma_{i j}}}{\|\psi\|_{0, \Gamma_{i j}}} \leq C \bar{h}_{j}\|u\|_{1, \Gamma_{i j}} .
\end{aligned}
$$

This and (3.6) gives (3.5).

The next lemma gives the approximation property for the space $X_{h}$.

Lemma 3.2. Let $u \in H_{0}^{1}(\Omega)$ and $\left.u\right|_{\Omega_{i}} \in H^{2}\left(\Omega_{i}\right)$ for all $i=1, \ldots, K$. Assume that the conditions (M.1), (A.2), (A.3), and (A.5) hold. Then there is a constant $C$ not depending on $\left\{\bar{h}_{i}\right\}_{i=1}^{K}$ such that

$$
\inf _{\chi \in X_{h}}\|u-\chi\|^{2} \leq C \sum_{i=1}^{K} \bar{h}_{i}^{2}\|u\|_{2, \Omega_{i}}^{2} .
$$

Proof. There is a discrete extension operator $E_{i j}: S_{h}^{0}\left(\Gamma_{i j}\right) \rightarrow X_{h, j}$ which satisfies (see, e.g., the construction in [32])

$$
E_{i j} v=\left\{\begin{array}{lll}
v & \text { on } & \Gamma_{i j} \\
0 & \text { on } & \partial \Omega_{j} \backslash \Gamma_{i j}
\end{array}\right.
$$

and

$$
\left\|E_{i j} v\right\|_{1, \Omega_{j}} \leq C\|v\|_{H_{00}^{1 / 2}\left(\Gamma_{i j}\right)}
$$


for all $v \in S_{h}^{0}\left(\Gamma_{i j}\right)$. Let $\bar{u} \in \widetilde{X}_{h}$ be the nodal finite element interpolation of $u$. Take $\chi=\bar{u}+\sum_{i j \in \mathcal{I}} E_{i j} \Pi_{i j}[\bar{u}] \in X_{h}$. By the triangle inequality, we get

$$
\|u-\chi\|^{2} \leq 2\left(\|u-\bar{u}\|^{2}+\left\|\sum_{i j \in \mathcal{I}} E_{i j} \Pi_{i j}[\bar{u}]\right\| \|^{2}\right) .
$$

The first term is bounded by standard finite element estimates. For the second, we note that condition (M.1) guarantees that $[\bar{u}] \in H_{00}^{1 / 2}\left(\Gamma_{i j}\right)$. Then, by Lemma 3.1 and (3.9),

$$
\left\|\sum_{i j \in \mathcal{I}} E_{i j} \Pi_{i j}[\bar{u}] \mid\right\|^{2} \leq C \sum_{i j \in \mathcal{I}}\|[\bar{u}]\|_{H_{00}^{1 / 2}\left(\Gamma_{i j}\right)}^{2} .
$$

Now

$$
\begin{aligned}
\|[u-\bar{u}]\|_{1, \Gamma_{i j}} & \leq\left\|\left.(u-\bar{u})\right|_{\Omega_{i}}\right\|_{1, \Gamma_{i j}}+\left\|\left.(u-\bar{u})\right|_{\Omega_{j}}\right\|_{1, \Gamma_{i j}} \\
& \leq C\left(\bar{h}_{i}^{1 / 2}+\bar{h}_{j}^{1 / 2}\right)|u|_{3 / 2, \Gamma_{i j}} .
\end{aligned}
$$

Similarly,

$$
\|[u-\bar{u}]\|_{0, \Gamma_{i j}} \leq C\left(\bar{h}_{i}^{3 / 2}+\bar{h}_{j}^{3 / 2}\right)|u|_{3 / 2, \Gamma_{i j}} .
$$

Interpolating between (3.11) and (3.12) gives

$$
\begin{aligned}
\|[\bar{u}]\|_{H_{00}^{1 / 2}\left(\Gamma_{i j}\right)} & =\|[u-\bar{u}]\|_{H_{00}^{1 / 2}\left(\Gamma_{i j}\right)} \\
& \leq C\left(\bar{h}_{i}^{1 / 2}+\bar{h}_{j}^{1 / 2}\right)^{1 / 2}\left(\bar{h}_{i}^{3 / 2}+\bar{h}_{j}^{3 / 2}\right)^{1 / 2}|u|_{3 / 2, \Gamma_{i j}} .
\end{aligned}
$$

Cauchy-Schwarz inequality and a trace theorem yields

$$
\begin{aligned}
\|[u-\bar{u}]\|_{H_{00}^{1 / 2}\left(\Gamma_{i j}\right)}^{2} & \leq C\left(\bar{h}_{i}^{2}+\bar{h}_{j}^{2}\right)|u|_{3 / 2, \Gamma_{i j}}^{2} \\
& \leq C\left(\bar{h}_{i}^{2}\|u\|_{2, \Omega_{i}}^{2}+\bar{h}_{j}^{2}\|u\|_{2, \Omega_{j}}^{2}\right) .
\end{aligned}
$$

Combining the above estimates and summing over $i j \in \mathcal{I}$ completes the proof of the lemma.

Remark 3.2. The conclusion of the previous lemma is still valid without (M.1) provided that the mortar triangulation $\mathcal{T}_{i}$ is globally quasi-uniform. For example, one could allow a face from the mortar triangulation $\mathcal{T}_{i}$ which intersects an interface $\Gamma_{i j}$ but is not completely contained in $\Gamma_{i j}$. This is illustrated in Figure 3 for a rectangular mesh and a similar situation occurs in our third numerical example in Section 5. Similar results have already been obtained in [11] in the case of continuous multipliers. We include this remark since it conforms to our numerical experiments.

In this case, $[\bar{u}]$ is no longer contained in $H_{00}^{1 / 2}\left(\Gamma_{i j}\right)$ and (3.10) does not make sense. The global quasi-uniformity condition implies that for all $v \in L^{2}\left(\Gamma_{i j}\right)$,

$$
\left\|\Pi_{i j} v\right\|_{H_{00}^{1 / 2}\left(\Gamma_{i j}\right)} \leq C \bar{h}_{j}^{-1 / 2}\left\|\Pi_{i j} v\right\|_{0, \Gamma_{i j}} .
$$

By (3.9), we have

$$
\left\|\sum_{i j \in \mathcal{I}} E_{i j} \Pi_{i j}[\bar{u}] \mid\right\|^{2} \leq C \sum_{i j \in \mathcal{I}}\left\|\Pi_{i j}[\bar{u}]\right\|_{H_{00}^{1 / 2}\left(\Gamma_{i j}\right)}^{2} .
$$




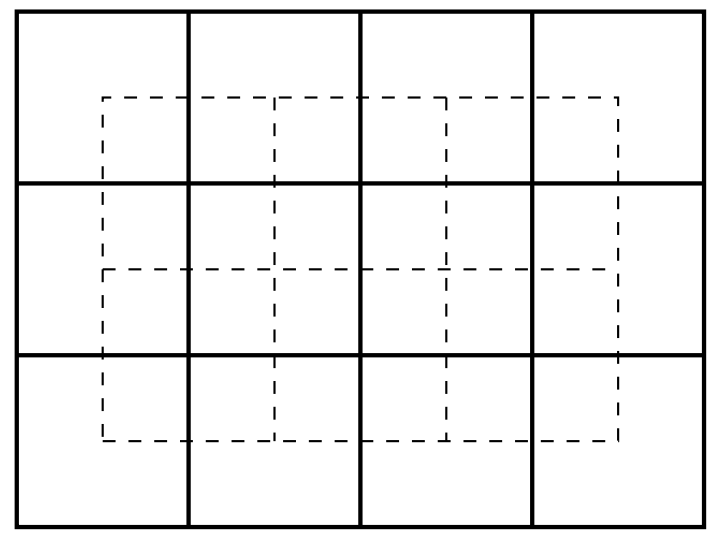

Figure 3. An example of a non-aligning face in rectangular mesh. The dotted lines depict the face $\Gamma_{i j}$ with the mesh inherited from the nonmortar subdomain $\Omega_{j}$, while the solid lines show the mesh from the mortar subdomain $\Omega_{i}$. None of the mortar boundary faces are contained in $\Gamma_{i j}$.

Then, for each ij $\in \mathcal{I}$, by the inverse inequality and the $L^{2}\left(\Gamma_{i j}\right)$-stability of $\Pi_{i j}$, we obtain

$$
\begin{gathered}
\left\|\Pi_{i j}[\bar{u}]\right\|_{H_{00}^{1 / 2}\left(\Gamma_{i j}\right)}^{2} \leq C \bar{h}_{j}^{-1}\|[\bar{u}]\|_{0, \Gamma_{i j}}^{2} \leq C \bar{h}_{j}^{-1}\left(\left\|\left.(u-\bar{u})\right|_{\Omega_{i}}\right\|_{0, \Gamma_{i j}}^{2}+\left\|\left.(u-\bar{u})\right|_{\Omega_{j}}\right\|_{0, \Gamma_{i j}}^{2}\right) \\
\leq C \bar{h}_{j}^{-1}\left(\bar{h}_{i}^{3}\|u\|_{2, \Omega_{i}}^{2}+\bar{h}_{j}^{3}\|u\|_{2, \Omega_{j}}^{2}\right) .
\end{gathered}
$$

Then,

$$
\left\|\Pi_{i j}[\bar{u}]\right\|_{H_{00}^{1 / 2}\left(\Gamma_{i j}\right)}^{2} \leq C\left(1+\frac{\bar{h}_{i}}{\bar{h}_{j}}\right)\left(\bar{h}_{i}^{2}\|u\|_{2, \Omega_{i}}^{2}+\bar{h}_{j}^{2}\|u\|_{2, \Omega_{j}}^{2}\right)
$$

and the conclusion of the lemma follows summing over $i j \in \mathcal{I}$.

We now prove Theorem 3.1.

Proof of Theorem 3.1. It follows from [7] that the bilinear form $\widetilde{A}(\cdot, \cdot)$ is coercive on the space of functions $v$ that are in $H^{1}\left(\Omega_{i}\right)$ in each $\Omega_{i}$, zero on the boundary $\partial \Omega$ and satisfy $\int_{\Gamma_{i j}}[v] d s=0$ on each interface $\Gamma_{i j}$. By (A.1), $X_{h}$ is contained in this space. Thus, by Strang's Lemma (see, e.g., [15]), we have

$$
\left\|\left|u-u_{h}\right|\right\| \leq C\left(\inf _{\chi \in X_{h}}\|u-\chi\|+\sup _{\eta \in X_{h} \backslash\{0\}} \frac{\left|\widetilde{A}\left(u-u_{h}, \eta\right)\right|}{\|\eta\|}\right) .
$$

Integration by parts gives

$$
\widetilde{A}\left(u-u_{h}, \eta\right)=\sum_{i j \in \mathcal{I}}\left(\frac{\partial u}{\partial n},[\eta]\right)_{\Gamma_{i j}}
$$

for all $\eta \in X_{h}$. Here, $n$ is the outward normal vector on $\Gamma_{i j}$ from the mortar subdomain $\Omega_{i}$. Now, for any $\gamma \in M_{h}\left(\Gamma_{i j}\right)$,

$$
\left(\frac{\partial u}{\partial n},[\eta]\right)_{\Gamma_{i j}}=\left(\frac{\partial u}{\partial n}-\gamma,[\eta]\right)_{\Gamma_{i j}} \leq C \bar{h}_{j}\left\|\frac{\partial u}{\partial n}\right\|_{1 / 2, \Gamma_{i j}}\|[\eta]\|_{1 / 2, \Gamma_{i j}} .
$$


Applying trace theorems, we obtain

$$
\left\|\frac{\partial u}{\partial n}\right\|_{1 / 2, \Gamma_{i j}} \leq C\|u\|_{2, \Omega_{j}}
$$

and

$$
\|[\eta]\|_{1 / 2, \Gamma_{i j}} \leq C\left(\|\eta\|_{1, \Omega_{i}}+\|\eta\|_{1, \Omega_{j}}\right) .
$$

Combining (3.15)-(3.17) and applying Cauchy-Schwarz inequality gives that

$$
\left|\widetilde{A}\left(u-u_{h}, \eta\right)\right| \leq C\|\| \eta \|\left(\sum_{i=1}^{K} \bar{h}_{i}^{2}\|u\|_{2, \Omega_{i}}^{2}\right)^{1 / 2} .
$$

The theorem follows from (3.14) and Lemma 3.2.

Remark 3.3. Suppose that for any $f \in L^{2}(\Omega)$, the solution $u$ to the problem (2.1) is in $H^{2}(\Omega)$ and satisfies

$$
\|u\|_{2} \leq C\|f\|_{0}
$$

If the mesh on $\Omega$ is globally quasi-uniform with size $h$, then conditions (M.1) and (A.1)(A.4) imply

$$
\left\|u-u_{h}\right\|_{0} \leq C h^{2}\|u\|_{2}
$$

The proof is based on Aubin-Nitsche duality argument and is omitted.

\section{EXAmples OF MULTIPLIER SPACES}

We consider three examples of multiplier spaces satisfying the conditions of the previous section. Specifically, we consider one dual basis example and two finite volume examples. The dual basis approach is the most interesting since it gives rise to the most efficient implementation and also extends to the case of locally quasi-uniform meshes. The finite volume multiplier spaces do not produce a diagonal mass matrix. However, these two spaces fit very well into the finite volume method for non-matching grids and lead to locally conservative approximations (see, e.g., [23]).

4.1. Dual basis multipliers. In this section, we consider a multiplier space defined in terms of a dual basis. We note that a dual basis approach for the mortar method was considered in the two dimensional case in [33] where it was suggested that although the method extends to three dimension, its extension was necessarily more complicated. According to [33], the complications were reflected in the quoted references [11] and [6] where restrictions on the triangles near the boundary were imposed. We will demonstrate here that the dual basis method extends to three dimensions without significant complication and any restrictions of the triangulation near the boundary even in the mesh refinement case.

We will define a dual basis method in terms of a map $I_{i j}$ which takes $S_{h}^{0}\left(\Gamma_{i j}\right)$ to the space of discontinuous functions which are linear when restricted to the triangles of $\mathcal{T}_{i j}$. Let $\tau$ be a triangle with vertices $\left\{y_{l} \mid l=1,2,3\right\}$ and $v_{l}$ denote the value of a function $\phi \in S_{h}^{0}\left(\Gamma_{i j}\right)$ at $y_{l}$. We define $I_{i j} \phi$ by the following rules:

1. If all three vertices of $\tau$ are in $\Gamma_{i j}$ then we set $I_{i j} \phi=w$ where $w$ is the linear function with values $w_{1}=3 v_{1}-v_{2}-v_{3}, w_{2}=3 v_{2}-v_{1}-v_{3}$, and $w_{3}=3 v_{3}-v_{1}-v_{2}$. 
2. If exactly one vertex ( $\left.\operatorname{say} y_{1}\right)$ of $\tau$ is on $\partial \Gamma_{i j}$, then we set $w_{1}=\left(v_{2}+v_{3}\right) / 2, w_{2}=$ $\left(5 v_{2}-3 v_{3}\right) / 2$, and $w_{3}=\left(5 v_{3}-3 v_{2}\right) / 2$.

3. If exactly one vertex ( $\operatorname{say} y_{1}$ ) of $\tau$ is in $\Gamma_{i j}$, then we set $w_{1}=w_{2}=w_{3}=v_{1}$.

4. If none of the vertices of $\tau$ are in $\Gamma_{i j}$ then we set $w_{1}=w_{2}=w_{3}=v_{l}$ where $v_{l}$ is value of $\phi$ at the interior vertex which is closest to the triangle.

Let $\left\{x_{l} \mid l=1, \ldots, n\right\}$ be the nodes in $\Gamma_{i j}$. We get a dual basis by defining $\psi_{l}=I_{i j} \phi_{l}$, for $l=1, \ldots, n$. In fact, it easily follows from the above definitions that $\left\{\psi_{l} \mid l=1, \ldots, n\right\}$ is linearly independent and satisfies $\left(\phi_{l}, \psi_{m}\right)=0$ whenever $l \neq m$. We define $M_{h}\left(\Gamma_{i j}\right)$ to be the span of $\left\{\psi_{l} \mid l=1, \ldots, n\right\}$.

From the above construction, it is clear that there is an integer $L$ (independent of the local mesh size) such that if $\tau \in \mathcal{T}_{i j}$ and $\phi \in S_{h}^{0}\left(\Gamma_{i j}\right)$ is 1 on every node which is within a distance of $L h_{\tau}$ of $\tau$, then $I_{i j} \phi$ equals one on $\tau$. This property implies that the space $M_{h}\left(\Gamma_{i j}\right)$ satisfies (A.1) and (A.4).

We next verify (A.3). Let $\phi=\sum d_{l} \phi_{l}$ be in $S_{h}^{0}\left(\Gamma_{i j}\right)$ and set $\psi=I_{i j} \phi=\sum d_{l} \psi_{l}$. Then,

$$
(\phi, \psi)_{\Gamma_{i j}}=\sum d_{l}^{2}\left(\phi_{l}, \psi_{l}\right)_{\Gamma_{i j}}
$$

Using the above definitions, it is easy to check that for any triangle $\tau$ with $x_{l}$ as a vertex,

$$
\left(\phi_{l}, \psi_{l}\right)_{\tau}=\frac{|\tau|}{3}
$$

Here $|\tau|$ denotes the area of the triangle $\tau$. The local quasi-uniformity of the mesh and (4.1) imply that

$$
\sum_{l} d_{l}^{2} h_{l}^{2} \leq C(\phi, \psi)_{\Gamma_{i j}}
$$

It is clear that the eigenvalues of the matrix

$$
\left(\begin{array}{ccc}
3 & -1 & -1 \\
-1 & 3 & -1 \\
-1 & -1 & 3
\end{array}\right)
$$

are positive and hence

$$
\int_{\tau}\left(I_{i j} \phi\right)^{2} d x \simeq \int_{\tau} \phi^{2} d x \simeq h_{\tau}^{2}\left(d_{l_{1}}^{2}+d_{l_{2}}^{2}+d_{l_{3}}^{2}\right)
$$

holds for triangles with interior vertices. Here $\left\{l_{k}\right\}$ are the indices for the vertices of $\tau$. Similar arguments can be applied to the remaining cases to show that

$$
\int_{\tau}\left(I_{i j} \phi\right)^{2} d x \simeq h_{\tau}^{2} \sum_{l_{j}} d_{l_{j}}^{2},
$$

where the sum above is over the indices of the nodes which determine $I_{i j} \phi$ on $\tau$. It follows that

$$
\|\phi\|_{0, \Gamma_{i j}}^{2} \simeq\left\|I_{i j} \phi\right\|_{0, \Gamma_{i j}}^{2}, \quad \text { for all } \phi \in S_{h}^{0}\left(\Gamma_{i j}\right) .
$$

Finally, by the local quasi-uniformity of the mesh,

$$
\|\phi\|_{0, \Gamma_{i j}}^{2} \simeq \sum_{l} d_{l}^{2} h_{l}^{2}, \quad \text { for all } \phi \in S_{h}^{0}\left(\Gamma_{i j}\right) .
$$


Combining the above estimates gives

$$
\|\phi\|_{0, \Gamma_{i j}} \leq C \frac{(\phi, \psi)_{\Gamma_{i j}}}{\|\psi\|_{0, \Gamma_{i j}}} .
$$

This verifies (A.3).

We finally verify (A.5). Let $\phi=\sum d_{l} \phi_{l} \in S_{h}^{0}\left(\Gamma_{i j}\right)$ and $\psi=\sum e_{l} \psi_{l} \in M_{h}\left(\Gamma_{i j}\right)$. Then

$$
(\hat{\phi}, \psi)_{\Gamma_{i j}}=\sum_{l=1}^{n} h_{l}^{-1} d_{l} e_{l}\left(\phi_{l}, \psi_{l}\right)=(\phi, \hat{\psi})_{\Gamma_{i j}}
$$

where $\hat{\psi}=\sum h_{l}^{-1} e_{l} \psi_{l}$. Now, by (4.2),

$$
\sum_{\tau \in \mathcal{T}_{i j}} h_{\tau}^{2}\|\hat{\psi}\|_{0, \tau}^{2} \leq C \sum_{l=1}^{n} h_{l}^{4}\left(h_{l}^{-1} e_{l}\right)^{2} \leq C\|\psi\|_{0, \Gamma_{i j}}^{2} .
$$

This is (A.5).

4.2. Finite volume multipliers. In the remainder of this section, we construct two examples of multiplier spaces involving piecewise constant functions defined over a partition of the interface $\Gamma_{i j}$. For both examples, we verify (A.1)-(A.4) so the abstract theory of the previous section can be applied when the mesh is globally quasi-uniform on each $\Gamma_{i j}$. Condition (A.5) is more difficult for these applications and may not hold without further assumptions on the meshes (more than locally quasi-uniform).

We start by splitting each triangle $\tau \in \mathcal{T}_{i j}$ into three quadrilaterals of equal area by connecting its medicenter with the midpoints of the sides of the triangle (see Figure 4). Thus, around each vertex $x_{l} \in \bar{\Gamma}_{i j}$ we take the quadrilaterals of all triangles having $x_{l}$ as a vertex. We denote this partition by $\mathcal{V}_{i j}$. Obviously, this partition contains volumes around all points on $\partial \Gamma_{i j}$ and the number of these volumes is greater than the dimension of the space $S_{h}^{0}\left(\Gamma_{i j}\right)$. We now reduce the number of the finite volumes to be equal to the number of the internal vertices in $\mathcal{T}_{i j}$ by the following construction.

1. If a triangle has all three vertices on $\partial \Gamma_{i j}$ then we attach this triangle to the adjacent one(s) through the common internal side(s).

2. If a triangle has exactly two vertices on $\partial \Gamma_{i j}$, we add this triangle and all those attached to it to the volume corresponding to the third vertex, which is in $\Gamma_{i j}$.

3. If a triangle has exactly one vertex, say $x_{1}$, on $\partial \Gamma_{i j}$, we split it into two parts by the median through $x_{1}$ and add the parts to the volumes corresponding to the internal vertices.

This forms a partition of $\Gamma_{i j}$ into disjoint volumes. This partition is denoted by $\mathcal{V}_{i j}^{0}$ (see Figure 4). Then the spaces of multipliers $M_{h}\left(\Gamma_{i j}\right)$ consists of all piecewise constant functions with respect to the partition $\mathcal{V}_{i j}^{0}$.

From the above construction, it is clear that the conditions (A.1) and (A.2) are satisfied.

We now verify (A.3). The characteristic functions $\left\{\chi_{l}\right\}$, corresponding to the volumes $\left\{V_{l} \in \mathcal{V}_{i j}^{0}\right\}$ form a basis for the space $M_{h}\left(\Gamma_{i j}\right)$. Let $\phi=\sum c_{l} \phi_{l}$ be in $S_{h}^{0}\left(\Gamma_{i j}\right)$ and set $\psi=\sum c_{l} \chi_{l}$. Then

$$
(\phi, \psi)_{\Gamma_{i j}}=\sum_{l, m} c_{l} c_{m} \int_{\Gamma_{i j}} \phi_{l} \chi_{m} d x=\sum_{\tau \in \mathcal{T}_{i j}} \sum_{l, m} c_{l} c_{m}\left(\phi_{l}, \chi_{m}\right)_{\tau} .
$$




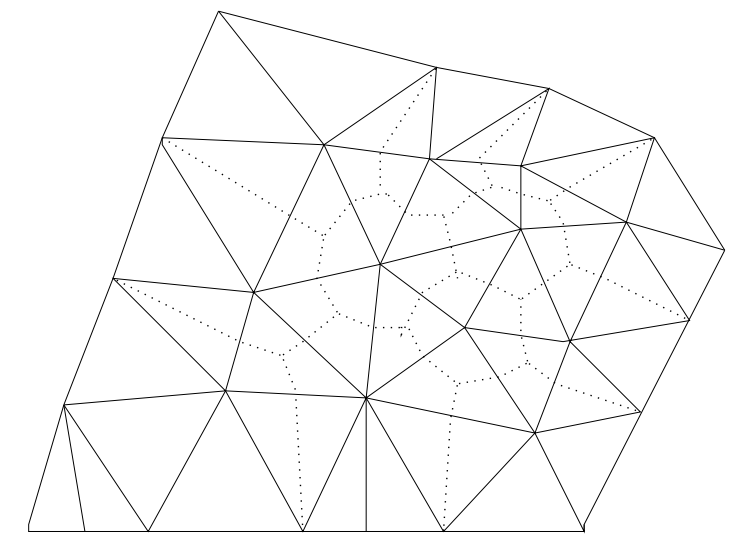

Figure 4. Finite element partition $\mathcal{T}_{i j}$ of the interface $\Gamma_{i j}$ and its finite volume (dual) partition $\mathcal{V}_{i j}^{0}$

We consider the element "mass" matrices with entries $\left(\phi_{l}, \chi_{m}\right)_{\tau}$ for $\phi_{l} \in S_{h}^{0}\left(\Gamma_{i j}\right), \chi_{m} \in$ $M_{h}\left(\Gamma_{i j}\right)$ and $\tau \in \mathcal{T}_{i j}$. Straightforward computations show that for an element $\tau$ with all vertices in $\Gamma_{i j}$, the element "mass" matrix is given by

$$
\frac{|\tau|}{108}\left(\begin{array}{ccc}
22 & 7 & 7 \\
7 & 22 & 7 \\
7 & 7 & 22
\end{array}\right)
$$

Similarly, if the finite element $\tau$ has exactly one vertex on $\partial \Gamma_{i j}$ then the corresponding "mass" matrix is the $2 \times 2$ matrix

$$
\frac{|\tau|}{12}\left(\begin{array}{ll}
3 & 1 \\
1 & 3
\end{array}\right)
$$

Finally, when $\tau$ has two vertices on $\partial \Gamma_{i j}$ then the matrix reduces to $|\tau| / 3$. Therefore, we have

$$
(\phi, \psi)=\sum_{\tau \in \mathcal{T}_{i j}} \sum_{l, m} c_{l} c_{m}\left(\phi_{l}, \chi_{m}\right)_{\tau} \geq \frac{1}{8} \sum_{\tau \in \mathcal{T}_{i j}}\left(c_{l_{1}}^{2}+c_{l_{2}}^{2}+c_{l_{3}}^{2}\right)|\tau| \simeq \sum_{l=1}^{n} h_{l}^{2} c_{l}^{2}
$$

where $l_{1}, l_{2}$, and $l_{3}$ are the indices of the vertices of the finite element $\tau$. These inequalities are valid even for triangles with vertices on $\partial \Gamma_{i j}$ provided that the corresponding $c_{l_{m}}$ 's are set to be zero. Moreover,

$$
\|\psi\|_{0, \Gamma_{i j}}^{2}=\sum_{V_{l} \in \mathcal{V}_{i j}^{0}} c_{l}^{2}\left|V_{l}\right| \simeq \sum_{l=1}^{n} h_{l}^{2} c_{l}^{2} \simeq\|\phi\|_{0, \Gamma_{i j}}^{2}
$$

Here $\left|V_{l}\right|$ denotes the area of $V_{l}$. Condition (A.3) follows immediately, combining the above inequalities.

Verification of (A.4) is also straightforward and follows immediately from Friedrichs' inequality on the domains in $V_{i j}^{0}$. 


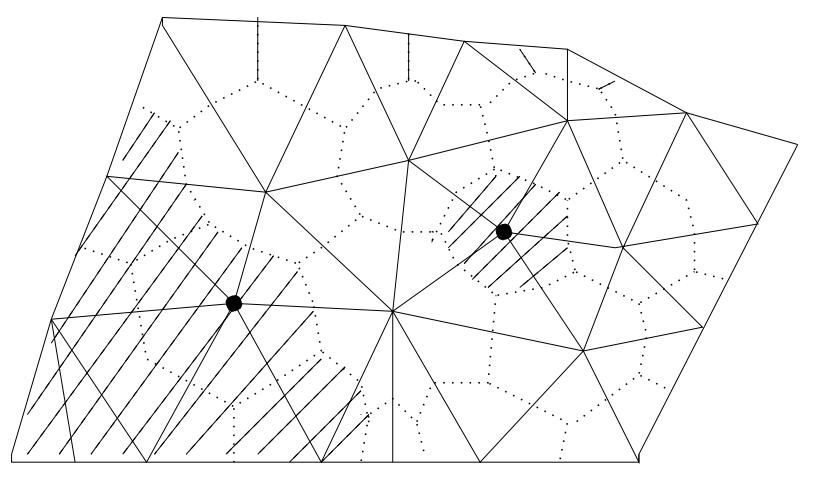

Figure 5. Examples of the support of the images $I_{i j} \phi$ of the nodal basis function $\phi \in S_{h}^{0}\left(\Gamma_{i j}\right)$

4.3. A second finite volume approach. We consider a second possibility for defining the mortar space based on the finite volume partition $\mathcal{V}_{i j}$ of the interface $\Gamma_{i j}$. This approach is similar to the approach of the dual basis discussed above. Namely, we define a map $I_{i j}$ which takes $S_{h}^{0}$ into the space of discontinuous functions which are constant when restricted to the volumes of $\mathcal{V}_{i j}$. The construction of the map $I_{i j}$ is based on a dual partition of the interface $\Gamma_{i j}$ used in the finite volume method. Below we construct such an operator and then define the space $M_{h}\left(\Gamma_{i j}\right)$ to be $I_{i j} S_{h}^{0}\left(\Gamma_{i j}\right)$.

For any $\phi=\sum_{l=1}^{n} c_{l} \phi_{l} \in S_{h}^{0}\left(\Gamma_{i j}\right)$, we set

$$
\psi=I_{i j} \phi(x)=\sum_{V_{l} \in \mathcal{V}_{i j}} d_{l} \chi_{l}(x)
$$

where $\chi_{l}(x)$ is the characteristic function of the finite volume $V_{l} \in \mathcal{V}_{i j}$, corresponding to the vertex $x_{l}$. The coefficients $d_{l}$ are determined in terms of the values of $\phi(x)$ in the following manner:

1. If $x_{l} \in \Gamma_{i j}$ then $d_{l}=c_{l}$.

2. If $x_{l} \in \partial \Gamma_{i j}$ and all its neighboring vertices are also on $\partial \Gamma_{i j}$ then we assign to $d_{l}$ the value of $\phi$ at the nearest internal vertex.

3. Finally, if $x_{l} \in \partial \Gamma_{i j}$ and has the internal vertices $x_{l_{1}}, \ldots, x_{l_{p}}$ (with $l_{p} \geq 1$ ) as its neighbors then we set

$$
d_{l}=\frac{\sum_{k=l_{1}}^{l_{p}} \alpha_{k l} c_{k}}{\sum_{k=l_{1}}^{l_{p}} \alpha_{k l}},
$$

where $\alpha_{k l}=\left|\tau_{1}\right|+\left|\tau_{2}\right|$ with $\tau_{1}$ and $\tau_{2}$ being the triangles sharing the edge connecting the vertices $x_{l}$ and $x_{k}$.

A basis for the resulting space $M_{h}\left(\Gamma_{i j}\right)=I_{i j} S_{h}^{0}\left(\Gamma_{i j}\right)$ is given by the images of the nodal basis function $\phi_{l} \in S_{h}^{0}\left(\Gamma_{i j}\right)$ (see Figure 5 for the support of these functions).

We now need to verify the conditions of the previous section. It is easy to see that $I_{i j} \phi=1$ for the function $\phi$ which is one on each node of $\Gamma_{i j}$. This verifies (A.1).

We verify (A.2) as follows. The dimension of $M_{h}\left(\Gamma_{i j}\right)$ is less than or equal to that of $S_{h}^{0}\left(\Gamma_{i j}\right)$ since $M_{h}\left(\Gamma_{i j}\right)$ is the image of $S_{h}^{0}\left(\Gamma_{i j}\right)$ under the linear map $I_{i j}$. For the other 
direction, let $\phi$ and $\psi$ be as in (4.6) above. Then,

$$
\left\|I_{i j} \phi\right\|_{0, \Gamma_{i j}}^{2}=\sum_{V_{l} \in \mathcal{V}_{i j}} d_{l}^{2}\left|V_{l}\right| \geq \sum_{l=1}^{n} c_{l}^{2}\left|V_{l}\right| \geq C\|\phi\|_{0, \Gamma_{i j}}^{2},
$$

which shows that the dimension of $I_{i j} S_{h}^{0}\left(\Gamma_{i j}\right)$ cannot be less than that of $S_{h}^{0}\left(\Gamma_{i j}\right)$. This verifies (A.2).

Next, we verify (A.3). We again let $\phi$ and $\psi$ be as in (4.6). Let $\tau$ be a triangle of $\Gamma_{i j}$, $\phi_{l}^{\tau}, l=1,2,3$, be the local linear nodal basis functions on $\tau$ and $\chi_{l}^{\tau}, l=1,2,3$, be the characteristic functions associated with the intersections of $\tau$ and the volumes in $\mathcal{V}_{i j}$. The element mass matrix with entries $\left(\phi_{l}^{\tau}, \chi_{m}^{\tau}\right)_{\tau}$ is given by $(4.5)$. It follows that

$$
(\phi, \psi)_{\tilde{\Gamma}_{i j} \cap \tau} \geq \frac{1}{8}\left(c_{l_{1}}^{2}+c_{l_{2}}^{2}+c_{l_{3}}^{2}\right)|\tau|
$$

where $\tilde{\Gamma}_{i j}=\cup_{x_{l} \in \Gamma_{i j}} V_{l}$. The above inequality is still valid when $\tau$ has nodes on $\partial \Gamma_{i j}$ as long as $c_{l_{m}}$ is defined to be zero for $x_{l_{m}} \in \partial \Gamma_{i j}$. Summing the above inequality gives

$$
(\phi, \psi)_{\tilde{\Gamma}_{i j}} \geq \frac{1}{8} \sum_{\tau \in \mathcal{T}_{i j}}\left(c_{l_{1}}^{2}+c_{l_{2}}^{2}+c_{l_{3}}^{2}\right)|\tau| \simeq \sum_{l=1}^{n} h_{l}^{2} c_{l}^{2} .
$$

Let $x_{l}$ be a boundary node. If all of its neighbors are on $\partial \Gamma_{i j}$, then $\phi$ vanishes on $V_{l}$ and thus the value of $\psi$ on $V_{l}$ does not affect $(\phi, \psi)_{\Gamma_{i j}}$. If $x_{l_{1}}, \ldots, x_{l_{p}}$ are the neighbors of $x_{l}$ which are in $\Gamma_{i j}$, then $d_{l}$ is given by (4.7) and an elementary computation gives

$$
(\phi, \psi)_{V_{l}}=\frac{7 d_{l}}{108} \sum_{k=l_{1}}^{l_{p}} \alpha_{k l} c_{k}=\frac{7 d_{l}^{2}}{108} \sum_{k=l_{1}}^{l_{p}} \alpha_{k l} \geq 0 .
$$

Combining this with (4.9) gives

$$
(\phi, \psi)_{\Gamma_{i j}} \geq C \sum_{l=1}^{n} h_{l}^{2} c_{l}^{2}
$$

It is easy to see that (4.3) holds for this space as well. Thus (A.3) follows from (4.10), (4.3) and (4.4).

From the above construction, it is clear that there is an integer $L$ (independent of the mesh) such that if $\tau \in \mathcal{T}_{i j}$ and $\phi \in S_{h}^{0}\left(\Gamma_{i j}\right)$ is one on every node which is within a distance of $L h_{\tau}$ of $\tau$, then $I_{i j} \phi$ equals one on $\tau$. As in the dual basis example, this property implies that the space $M_{h}\left(\Gamma_{i j}\right)$ satisfies (A.4).

\section{NUMERICAL RESULTS}

In this section, we present three numerical examples. In the first example, both the subdomain partition and the triangulation align, while in the others neither does. Subdomain partitions and mesh structure of the first two examples are illustrated in Figures 6-8 and those of the third in Figure 9. The non-matching grids at some of the interfaces for the first two examples are illustrated in Figures 7 and 8, respectively.

In each example, trilinear finite elements on meshes of rectangular parallelepipeds and the corresponding dual basis multiplier are used. To construct this multiplier space for our rectangular mesh, which in fact is the tensor product of the two dimensional dual basis multiplier considered in [33], we use a straightforward extension of the techniques 

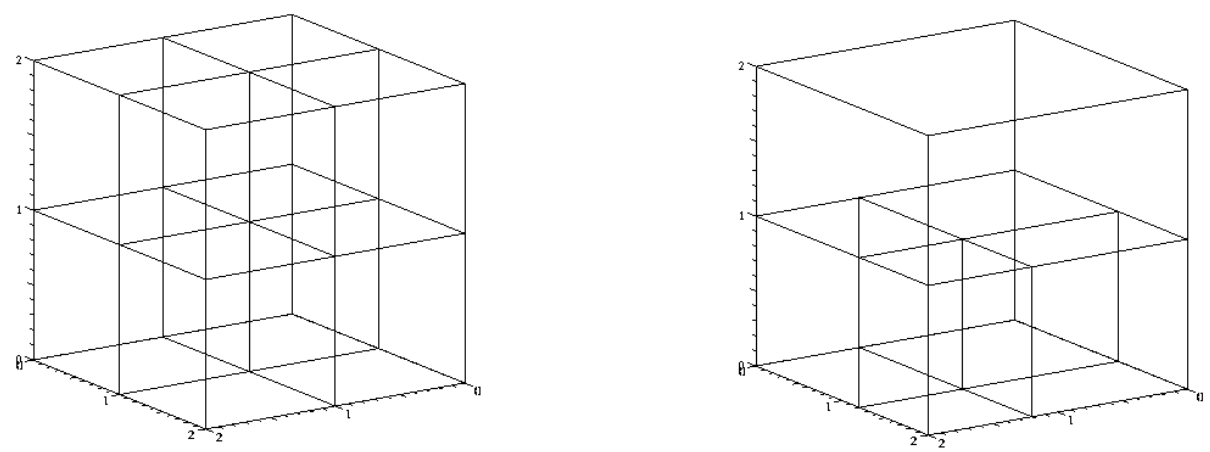

FiguRE 6. Subdomain partitions for the first (left) and second (right) examples.
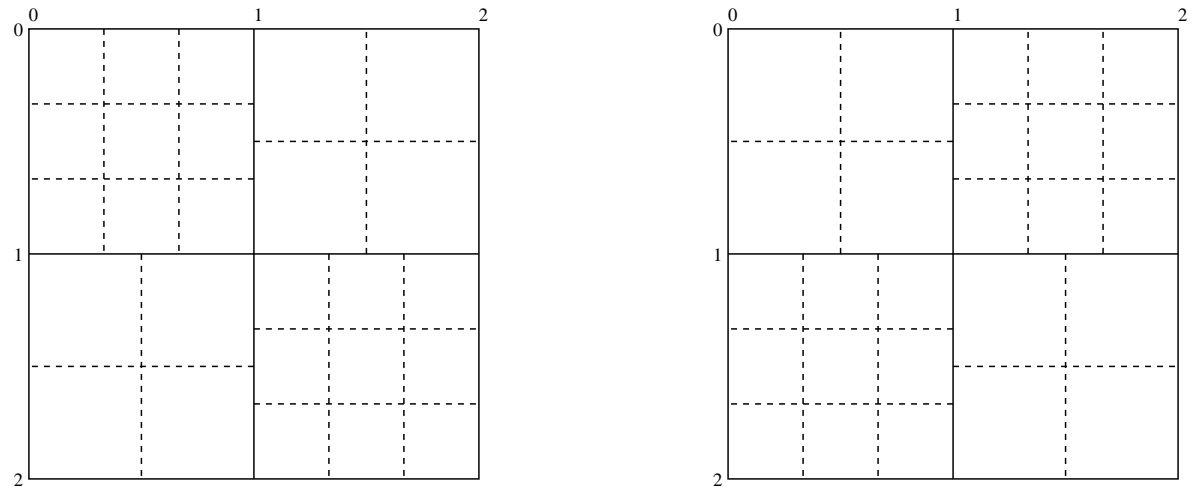

FiguRE 7. Initial mesh for the first example at $z=1$ for the upper (left) and the lower (right) subdomains. Solid lines denote subdomain boundaries and dashed lines the mesh.
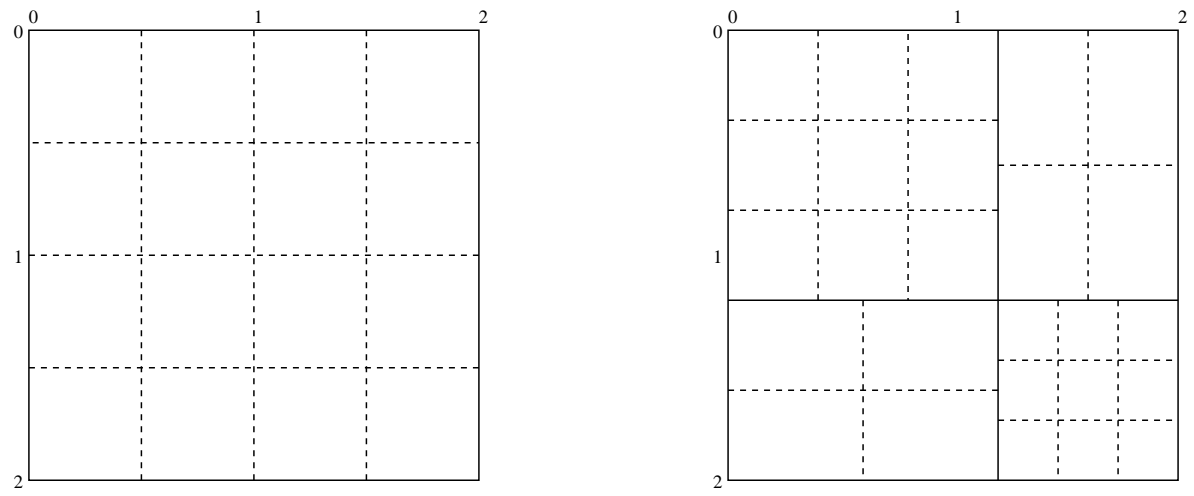

FiguRE 8. Initial mesh for the second example at $z=1$ for the upper subdomain (left) and the 4 lower (right) subdomains. Solid lines denote subdomain boundaries and dashed lines the mesh.

developed in Section 4.1 for general triangular mesh. Even though the theory given in the previous sections was for tetrahedral meshes, it extends to the approximation described above without difficulty. 


\begin{tabular}{|c|c|c|c|}
\hline level & number of elements & $\|\cdot\|_{0}$-error & $\|\cdot|\||$-error \\
\hline 1 & 140 & $5.14 \mathrm{e}-2$ & $4.74 \mathrm{e}-1$ \\
2 & 1120 & $1.28 \mathrm{e}-2$ & $2.36 \mathrm{e}-1$ \\
3 & 8960 & $3.16 \mathrm{e}-3$ & $1.17 \mathrm{e}-1$ \\
4 & 71680 & $7.87 \mathrm{e}-4$ & $5.84 \mathrm{e}-2$ \\
\hline
\end{tabular}

TABLE 1. Error behavior for the first example.

\begin{tabular}{|c|c|c|c|}
\hline level & number of elements & $\|\cdot\|_{0 \text {-error }}$ & $\|\cdot \cdot\|||$-error \\
\hline 1 & 84 & $6.30 \mathrm{e}-2$ & $5.58 \mathrm{e}-1$ \\
2 & 672 & $1.59 \mathrm{e}-2$ & $2.73 \mathrm{e}-1$ \\
3 & 5376 & $3.98 \mathrm{e}-3$ & $1.35 \mathrm{e}-1$ \\
4 & 43008 & $9.95 \mathrm{e}-4$ & $6.74 \mathrm{e}-2$ \\
\hline
\end{tabular}

TABLE 2. Error behavior for the second example.

The first two examples deal with a Dirichlet problem $(2.1)$ on $\Omega=(0,2)^{3}$. The error behavior in the norms $\|\cdot\|_{0}$ and $\|\mid \cdot\|$ for the known solution

$$
u(x, y, z)=e^{-(x-1 / 2)^{2}-(y-1)^{2}-(z-3 / 2)^{2}} x y z(2-x)(2-y)(2-z)
$$

is reported in Tables 1 and 2. At each level after the first, a finer mesh is obtained by partitioning each element into 8 identical ones. In both examples, we observe second order convergence in the $\|\cdot\|_{0}$-norm and first order convergence in the $\||\cdot \||-$-norm.

In our third example, a linear elasticity problem is considered. We solve for $u=$ $\left(u_{1}, u_{2}, u_{3}\right)$ satisfying, for each $j=1,2,3$,

$$
\begin{aligned}
\sum_{i=1}^{3} \frac{\partial}{\partial x_{i}} \sigma_{i j}(u) & =0 \quad \text { in } \Omega \\
u_{j} & =0 \quad \text { on } \Gamma_{D} \\
\sum_{i=1}^{3} \sigma_{i j} n_{i} & =f_{j} \text { on } \Gamma_{N},
\end{aligned}
$$

where, for each $i, j=1,2,3$,

$$
\begin{aligned}
\sigma_{i j}(u) & =2 \mu \varepsilon_{i j}(u)+\lambda \delta_{i j} \nabla \cdot u, \\
\varepsilon_{i j}(u) & =\frac{1}{2}\left(\frac{\partial u_{i}}{\partial x_{j}}+\frac{\partial u_{j}}{\partial x_{i}}\right),
\end{aligned}
$$

with $\mu=8.2$ and $\lambda=10\left(\mathrm{~kg} / \mathrm{cm}^{3}\right)$, the Lamé coefficients for steel. Here, $f=\left(f_{1}, f_{2}, f_{3}\right)$ is given by $f_{1}=f_{2} \equiv 0$ and

$$
f_{3}= \begin{cases}-0.35 & \text { if } 22 \leq y \leq 28 \\ 0 & \text { otherwise }\end{cases}
$$

Our computational domain $\Omega$ in this example is an I-beam contained in $(0,50) \times$ $(0,10) \times(0,13)$, constructed by combining 3 plates, one at the top, another in the middle, and the other at the bottom. Each plate makes a subdomain, as shown in the 

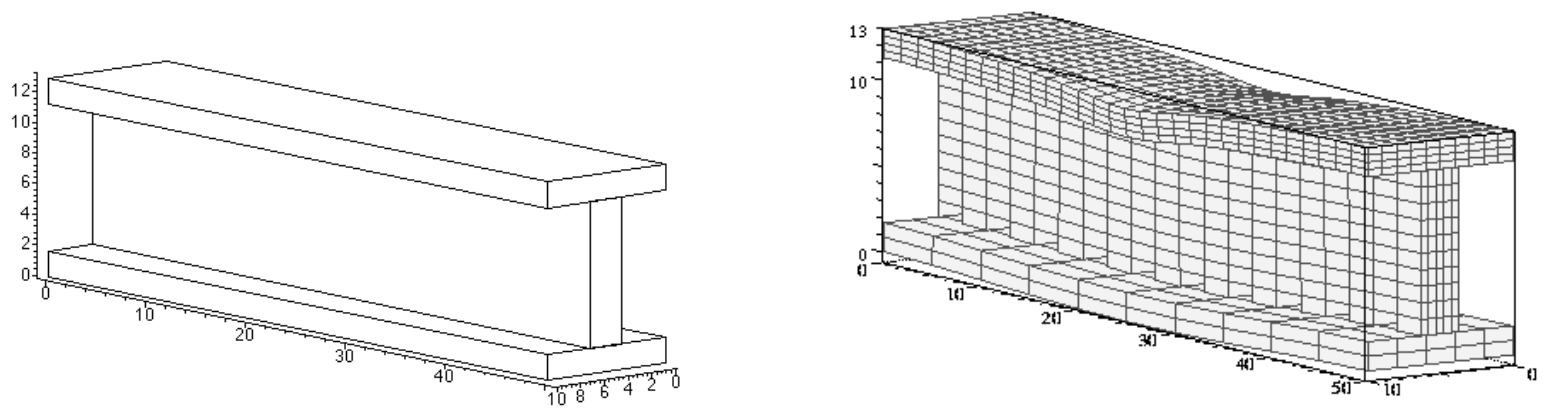

Figure 9. Subdomain partition (left) and the computed solution with the mesh (right) for the I-beam example.

left picture of Figure 9. This yields a non-aligning subdomain partition. Then each subdomain is meshed independently of the others, resulting in a non-aligning global mesh. We compute the displacement $u$ when the beam is fixed at $\Gamma_{D}$, the two I-shaped ends, and a constant vertical force $f$ is applied to $\Gamma_{N}$, a central region of the top surface. The resulting deformation of the beam, along with the mesh, is presented in the right image of Figure 9.

\section{REFERENCES}

[1] Y. Achdou, Yu. Kuznetsov, and O. Pironneau, Substructuring preconditioners for the $Q_{1}$ mortar element method, Numer. Math., 71 (1995), pp. 419-449.

[2] Y. Achdou, Y. Maday, and O. Widlund, Iterative substructuring preconditioners for mortar element methods in two dimensions, SIAM J. Numer. Anal., 36 (1999), pp. 551-580.

[3] G. Anagnostou, Y. Maday, C. Mavriplis, and A. Patera, On the mortar element method: generalization and implementation, in Proc. Third Int. Conf. on Domain Decomposition Methods for PDEs, T. Chan et al., eds., SIAM Philadelphia, 1990.

[4] T. Arbogast and I. Yotov, A non-mortar mixed finite element method for elliptic problems on nonmatching multi-block grids, Comp. Meth. in Appl. Mech. and Engng., 149 (1997), pp. 255-265.

[5] F. Ben Belgacem, The mortar finite element method with Lagrange multiplier, Numer. Math., 84 (1999), pp. 173-197.

[6] F. Ben Belgacem and Y. Maday, The mortar element method for three dimensional finite elements, $\mathrm{M}^{2}$ AN Math. Model. Numer. Anal., 31 (1997), pp. 289-302.

[7] C. Bernardi, Y. Maday, and A. T. Patera, Domain Decomposition by the mortar element method, in Asymptotic and Numerical Methods for Partial Differential Equations with Critical Parameters, H.G. Kaper and M. Garbey, eds., Kluwer Academic Publishers (1993), pp. 269-286.

[8] C. Bernardi, N. Debit, and Y. Maday, Coupling finite element and spectral methods: First results, Math. Comp. 54 (1990), pp. 21-39.

[9] C. Bernardi, Y. Maday, and A. Patera, A new non conforming approach to domain decomposition: The mortar element method, in Nonlinear Partial Differential Equations and Their Applications, H. Brezis and J. L. Lions, eds., Pitman Research Notes in Math. Series 299, Longman 1994, pp. 13-51 (appeared in 1989 as Technical Report).

[10] C. Bernardi, Y. Maday, and G. Sacchi-Landriani, Nonconforming matching conditions for coupling spectral finite element methods, Appl. Numer. Math., 54 (1989), pp. 64-84.

[11] D. Braess and W. Dahmen, Stability estimates of the mortar finite element method for 3dimensional problems, East-West J. Numer. Math., 6 (1998), pp. 249-264.

[12] D. Braess, W. Dahmen, and C. Wieners, A multigrid algorithm for the mortar finite element method, SIAM J. Numer. Anal., 37 (1999), pp. 48-69. 
[13] J. H. Bramble, J. E. Pasciak, and O. Steinbach, On the stability of the $L^{2}$ projection in $H^{1}(\Omega)$, Math. Comp. (to appear).

[14] J. H. Bramble and J. Xu, Some estimates for weighted $L^{2}$-projections, Math. Comp. 56 (1991), pp. $463-476$.

[15] S. Brenner and L. R. Scott, The Mathematical Theory of Finite Element Methods, Springer-Verlag, 1994.

[16] C. Carstenen, Merging the Bramble-Pasciak-Steinbach and the Crouzeix-Thomeé criterion for $H^{1}$ stability of the $L^{2}$ projection onto finite element spaces, Math. Comp. (to appear).

[17] M. Casarin and O.B. Widlund, A hierarchical preconditioner for the mortar finite element method, Electr. Trans. Numer. Anal., 4 (1996), pp. 75-88.

[18] T. F. Chan, R. Glowinski, J. Periaux, and O. B. Widlund, eds., Third Int. Symposium on Domain Decomposition Methods for Partial Differential Equations, SIAM, Philadelphia, PA, 1990.

[19] T. F. Chan, R. Glowinski, J. Periaux, and O. B. Widlund, eds., Domain Decomposition Methods, SIAM, Philadelphia, PA, 1989.

[20] P. Clément, Approximation by finite element functions using local regularization, RAIRO, Anal. Numér. 9 (1975), no. R-2, pp. 77-84.

[21] M. Crouzeix and V. Thomeé, The stability in $L_{p}$ and $W_{p}^{1}$ of the $L^{2}-$ projection onto finite element function spaces, Math. Comp. 48 (1987), pp. 521-532.

[22] M. Dorr, On the discretization of inter-domain coupling in elliptic boundary-value problems via the p-version of the finite element method, in Domain Decomposition Methods, T. F. Chan, R. Glowinski, J. Periaux, and O. B. Widlund, eds., SIAM, 1989. pp. 17-37.

[23] R. E. Ewing, R. D. Lazarov, T. Lin, and Y. Lin, The mortar finite volume element methods and domain decompositions, East-West J. Numer. Math. 8 (2000), pp. 93-110.

[24] R. Glowinski, G. H. Golub, G. A. Meurant, and J. Periaux, eds., First Int. Symposium on Domain Decomposition Methods for Partial Differential Equations, SIAM, Philadelphia, PA, 1988.

[25] R. Glowinski, Yu. A. Kuznetsov, G. A. Meurant, and J. Periaux, eds., Fourth Int. Symposium on Domain Decomposition Methods for Partial Differential Equations, SIAM, Philadelphia, PA, 1991.

[26] J. Gopalakrishnan, On the Mortar Finite Element Method, Ph.D. thesis, 1999, Texas A\&M University.

[27] J. Gopalakrishnan and J. E. Pasciak, Multigrid for the mortar finite element method, SIAM J. Numer. Anal., 37 (2000), pp. 1029-1052.

[28] P. Grisvard, Elliptic Problems in Nonsmooth Domains, Monographs and Studies in Mathematics \#24, Pitman, 1985.

[29] Yu. Kuznetsov and M. F. Wheeler, Optimal order substructuring preconditioners for mixed finite element methods on non-matching grids, East-West J. Numer. Math., 3 (1995), pp. 127-143.

[30] R. D. Lazarov, J. E. Pasciak, and P. S. Vassilevski, Coupling mixed method and finite volume discretizations of convection-diffusion-reaction equations on non-matching grids, Proc. Second Symposium Finite Volumes for Complex Applications, Duisburg, Germany, July 19-22, 1999, Hermes, pp. $51-68$.

[31] J. L. Lions and E. Magenes, Non-homogeneous Boundary Value Problems and Applications, volume I, Springer-Verlag, 1972.

[32] P. Seshaiyer and M. Suri, Uniform hp convergence results for the mortar finite element method, Math. Comp., 69 (2000), pp. 521-546.

[33] B. I. Wohlmuth, A mortar finite element method using dual spaces for the Lagrange multiplier, Preprint 407, Universtät Augsburg, 1998 (submitted).

[34] B. I. Wohlmuth and R. H. Krause, Mulitigrid methods based on the unconstrained product space arising from mortar finite element discretizations, (preprint). 
Dept. of Mathematics, Texas A \& M University, College Station, TX 77843, USA. E-mail address: cskim@math.tamu.edu

Dept. of Mathematics, Texas A \& M University, College Station, TX 77843, USA. E-mail address: lazarov@math.tamu.edu

Dept. of Mathematics, Texas A \& M University, College Station, TX 77843, USA. E-mail address: pasciak@math.tamu.edu

Center for Applied Scientific Computing, Lawrence Livermore National Laboratory, P. O. Box 808, L-560, Livermore, CA 94551, U.S.A.

E-mail address: panayot@llnl.gov 